\title{
EELS Analyses of Gaseous Atmosphere and Heated Specimen in an ETEM
}

Toshie Yaguchi ${ }^{1}$, Hiroyuki Kobayashi ${ }^{1}$, Akira Watabe ${ }^{1}$, and Takeo Kamino ${ }^{1,2}$

1. Advanced Microscope Systems Design Dept., Hitachi High-Technologies Corp., Ibaraki, Japan

2. Fuel Cell Nanomaterials Research Center, Yamanashi University, Kofu, Japan

Since we have developed a specimen heating holder equipped with a gas injector for use with high resolution environmental TEMs, our study was mainly focused on structural changes of nano materials heated in various gaseous atmospheres at elevated temperatures [1-3]. Most studies were carried out on the assumption that the composition of the gas surrounding the specimen is the same as that of introduced gas.

Recently, feasibility of performing analyses of gas in the environmental TEM by electron energy-loss spectroscopy (EELS) has been investigated [4]. This paper presents our study on the possibility of EELS analysis of gas around the specimen and a heated specimen in the gas environment in our ETEM system.

The instrument used in the study was an H-9500 environmental TEM equipped with a Gatan GIF system. Figure 1 shows a schematic illustration of the basic construction of the specimen heating holder. In this specimen holder, the gas injector with inner diameter of $0.5 \mathrm{~mm}$ is located at the distance of $1 \mathrm{~mm}$ from specimen mounted on the heating element. Figure 2 shows the pressure distribution around the specimen measured using a micro pressure gauge attached to the specimen holder. In this construction, the reaction gas is directly introduced to the specimen by means of the gas injector with high pressure so that a gaseous atmosphere is formed locally in the vicinity of the specimen.

The EELS analyses were carried out at $300 \mathrm{kV}$ using single crystal $\mathrm{LaB}_{6}$ thermionic source. The energy resolution of the system at $300 \mathrm{kV}$ is $1.4 \mathrm{eV}$ (figure 3). The EELS analyses were carried out in image mode with a collection semi-angle of $4 \mathrm{mrad}$.

The gases of $\mathrm{H}_{2}, \mathrm{~N}_{2}, \mathrm{O}_{2}, \mathrm{CO}$ and air were separately introduced to the specimen chamber with the maximum pressure of $1 \mathrm{~Pa}$ and nano-material specimens such as electrocatalysts were heated to $800{ }^{\circ} \mathrm{C}$. Dynamic observation of structural changes of the heated specimens using CCD-camera system and the EELS analyses of the gas and the heated specimen were carried out alternately.

Figure 4 shows an example of simultaneous EELS analysis of heated specimen (Ketjen Black) and introduced gas with the estimated pressure of $5 \mathrm{~Pa}$. K-edges of $\mathrm{N}_{2}$ and carbon are clearly demonstrated.

Figure 5 shows low-loss spectra recorded before (a) and 30 seconds after (b) stopping the introduction of $\mathrm{H}_{2}$ gas with the estimated pressure of $5 \mathrm{~Pa}$.

The results of the in situ EELS analyses of gases show that the gas atmosphere of the specimen area changes within 30 seconds after replacement of introduced gas which is 2-5 times faster than that of our conventional environmental TEMs and it give evidence of the advantage of the specimen heating holder consists of a gas injector inside the holder.

References:

[1] Kamino T, et al, J. Electron Microsc.54, (2005) pp.497-503.

[2] Kishita K, et al, J. Electron Microsc.58, (2009) pp.331-339.

[3] Yaguchi T, et al, J. Electron Microsc. 60, (2011) pp.217-225.

[4] P.A Crozier, et al, Microsc.Microanal 16 (Suppl.2) (2010) pp.298-299. 


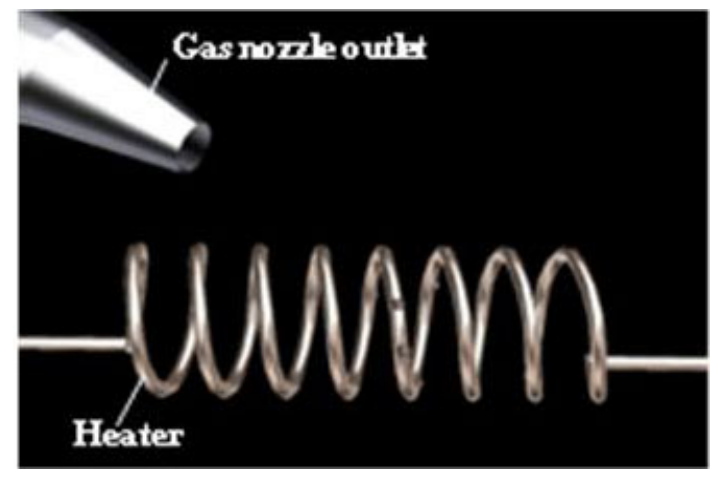

Figure 1. Schematic illustration of the principle of the specimen holder.

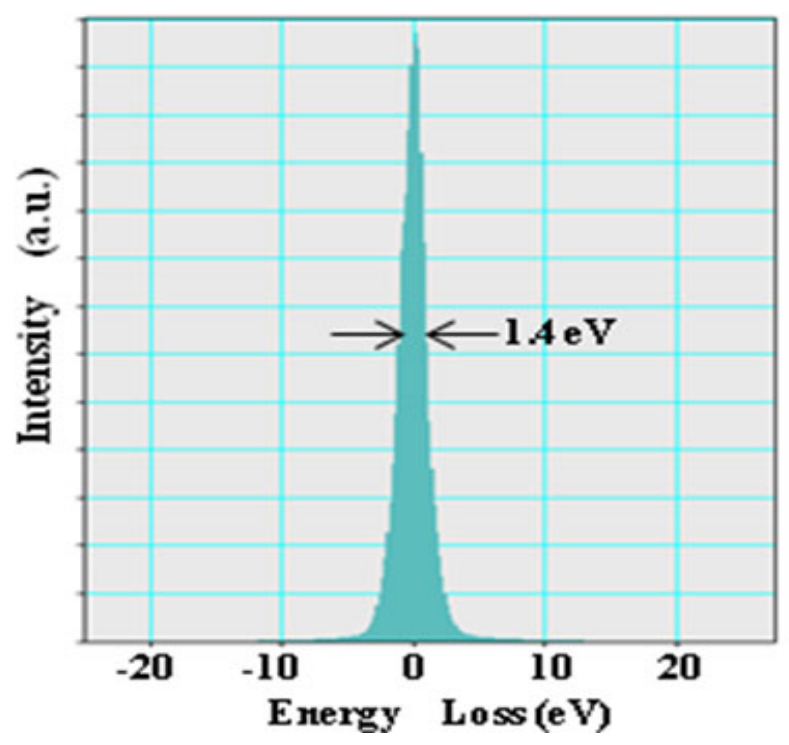

Figure 3. Energy resolution of the system at $300 \mathrm{kV}$ in vacuum.

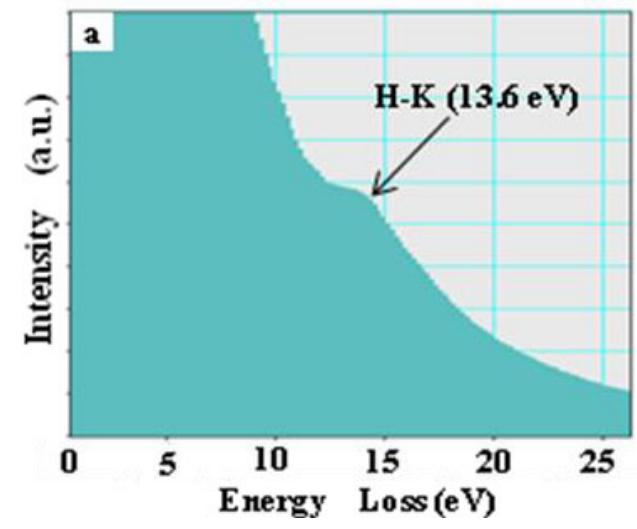

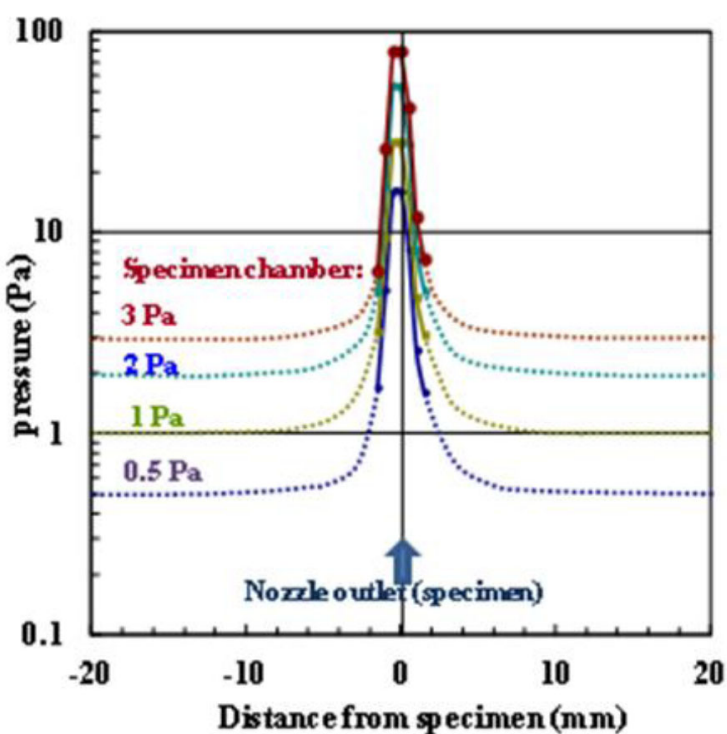

Figure 2. Measured pressure distribution around the specimen position.

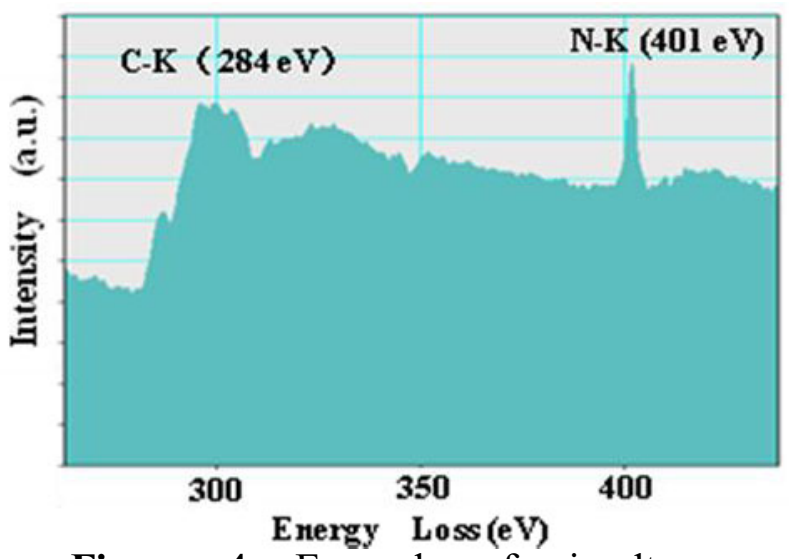

Figure 4. Example of simultaneous EELS analysis of a heated specimen (Ketjen Black) and introduced gas.

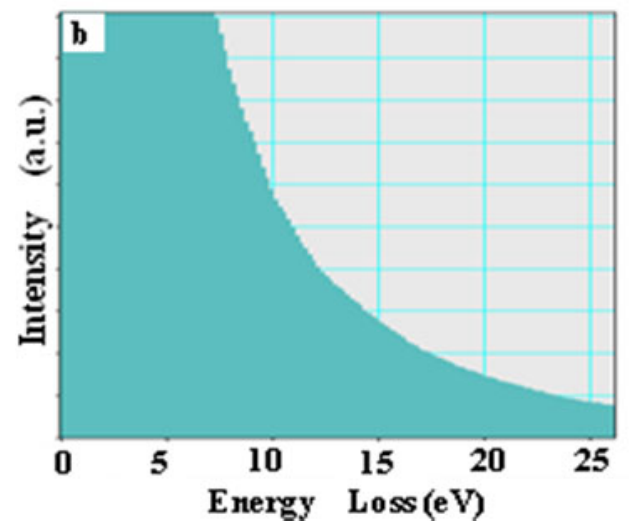

Figure 5. low-loss spectra recorded before (a) and 30 seconds after (b) stopping the introduction of $\mathrm{H}_{2}$ with the estimated pressure of $5 \mathrm{~Pa}$. 